\title{
Mediastinal Bronchial Artery Aneurysm Mimicking a Subcarinal Tumor
}

\author{
Rie Tsuboi Masahide Oki Hideo Saka \\ Department of Respiratory Medicine, Nagoya Medical Center, Nagoya, Japan
}

An 82-year-old male with bronchiectasis was admitted to our hospital because of hemoptysis. A chest computed tomography $(\mathrm{CT})$ showed a round mass measuring $33 \mathrm{~mm}$ in diameter under the tracheal bifurcation (fig. 1). To distinguish between an artery and a tumor, a contrast-

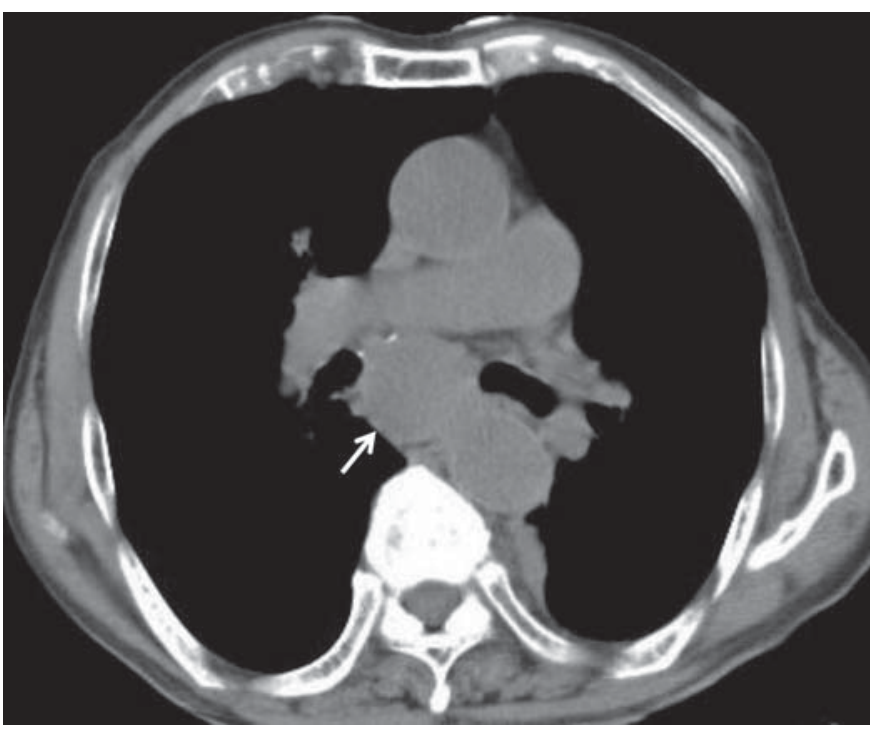

Fig. 1. Chest CT showing a mass (arrow) in the subcarinal region.

\section{KARGER}

Fax +4161306 1234

E-Mail karger@karger.ch

www.karger.com
(C) 2011 S. Karger AG, Basel

$0025-7931 / 11 / 0882-0207 \$ 38.00 / 0$

Accessible online at:

www.karger.com/res enhanced CT was performed which revealed a homogeneously enhanced mass that indicated a mediastinal bronchial artery aneurysm (fig. 2). For further evaluation and endovascular treatment, bronchial arteriography was performed and confirmed a mediastinal bronchial

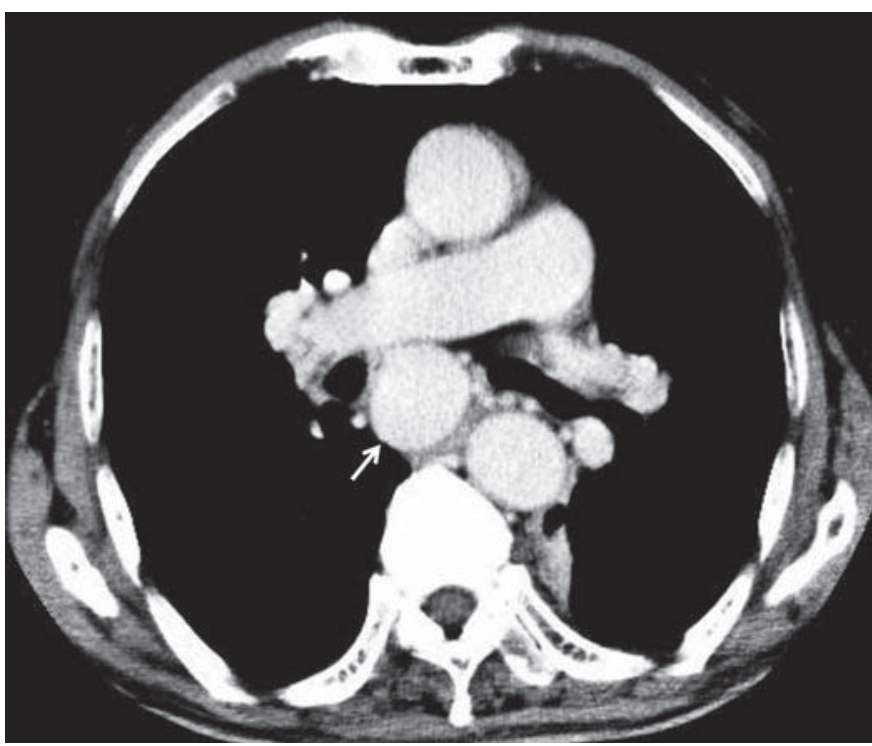

Fig. 2. Contrast-enhanced CT showing the enhanced mass (arrow) suggesting a bronchial artery aneurysm. 


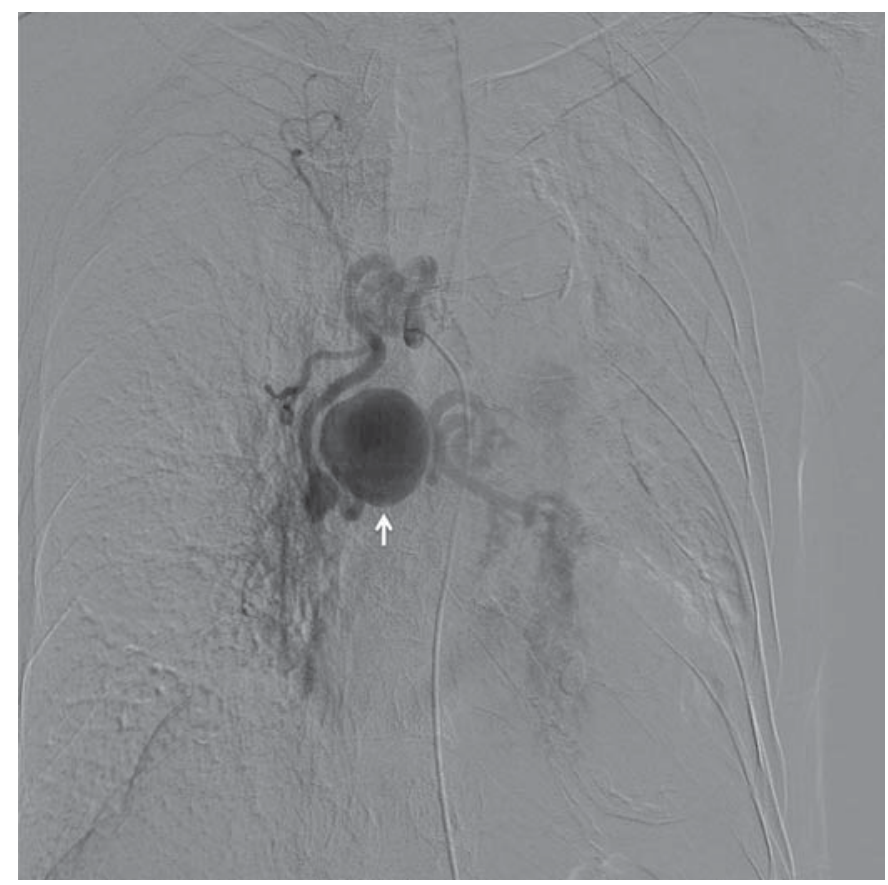

Fig. 3. Bronchial arteriography showing a mediastinal bronchial artery aneurysm (arrow) arising from the left bronchial artery.

artery aneurysm arising from the left bronchial artery; CT revealed it to be a subcarinal mass (fig. 3). Coil embolization was not technically feasible because of the large size of the aneurysm and the difficulty of threading the serpentine, narrow feeding artery with the microcathe-

ter. Hence, embolization using a gelatin sponge was performed. The patient was reluctant to undergo further surgical treatment and was discharged 11 days thereafter as the hemoptysis was stopped.

Bronchial artery aneurysms, which can be found in the lung and mediastinum, are classified as congenital or secondary; the latter are caused by inflammation or hyper bloodstream conditions, such as bronchiectasis, infection, or cancer [1]. Mediastinal bronchial artery aneurysms sometimes have a radiological appearance similar to that of mediastinal tumors [1]. Although bronchial artery aneurysms may be rare, pulmonologists and bronchoscopists should be familiar with the disease. While performing an examination, one can mistake an aneurysm for a tumor and instead perform a transbronchial needle aspiration, which has been reported to cause significant bleeding [2].

References
1 Tanaka K, Ihaya A, Horiuci T, Morioka K, Kimura T, Uesaka T, Sasaki M, Uchinami M, Tsuda T, Yamada N, Li W, Hirai S, Tanabe S, Okubo Y, Tanaka S: Giant mediastinal bronchial artery aneurysm mimicking benign esophageal tumor: a case report and review of 26 cases from literature. J Vasc Surg 2003; 38:1125-1129.

2 Gibbs PM, Hami A: Pulmonary arterial aneurysm presenting as an endobronchial mass. Thorax 1995;50:1013-1014. 